\title{
Invasive mucormycosis [zygomycosis] and osteomyelitis affecting maxilla: A rare case report
}

\author{
J. B. Garde ${ }^{1}$, Harshad Bhagwat ${ }^{2}$, Ashvini Vadane ${ }^{3, *}$, Sharmishtha Gaikwad ${ }^{4}$ \\ ${ }^{1}$ HOD \& Guide, ${ }^{2}$ Reader \& Guide, ${ }^{3,4}$ Postgraduate Student, ${ }^{1-3}$ Dept. of Oral and Maxillofacial Surgery and Implantology, ${ }^{4}$ Dept. \\ of Prosthodontics, M A Rangoonwala College Of Dental Science \& Research Centre, Pune, Maharashtra, India
}

*Corresponding Author:

Email: drashvinivadane@gmail.com

\begin{abstract}
"Mucormycosis" is an opportunistic infection caused by fungi belonging to the Mucorales order , and was first described by "Paultauf" in $1885 .{ }^{3}$ It is the third invasive mycosis after candidiasis and aspergillosis and having high mortality of 70-100\%, it accounts for $8.3-13 \%$ of all fungal infections. ${ }^{1}$ Immunocompromised host is typically get affected by mucormycosis but healthy patients with some form of trauma and patients who are having history of environmental exposure can develop infection as well. ${ }^{4}$ The present article reports a case of "right side maxillary mucormycosis" which was successfully managed surgically in M.A. Rangoonwala dental college, Pune.
\end{abstract}

Keywords: Mucormycosis, Fungal infection, Opportunistic infection.

\section{Introduction}

Mucormycosis is the third most common fungal infection. It is an invasive opportunistic infection. In mucormycosis patients, mortality rate can reach upto $100 \%$ depending upon the form of mucormycosis and patient's underlying disease. ${ }^{1}$

This article reports one case of "maxillary mucormycosis". This case was managed surgically and was rehabilitated prosthodontically in M. A. Rangoonwala Dental College, Pune.

\section{Case Report}

A 52 years old male patient visited department of "oral \& maxillofacial surgery" of M.A. Rangoonwala dental college, Pune with the chief complaints of severe headache, watering from eyes and swelling \& tingling numbness over right side of face. Patient is a known case of diabetes mellitus and hypertension since 11 years and under medication for that, he is also having history of "ischemic heart disease" and is under medication for the same since 6 years. Before visiting our institute, patient had visited local hospital and had been diagnosed for facial cellulitis with maxillary sinusitis and right side facial palsy. The "endoscopic sinus surgery" by that local hospital diagnosed "right maxillary mucormycosis".

When patient visited our institute, he was suffering from swelling on right side of face, extending from ala of nose to pretragal region. Radiographic examination revealed "right maxillary sinusitis with osteomyelitis". For the management of this Pathology, we planned for "right side Caldwell-Luc surgery" and debridement under general anaesthesia. After surgery, specimen was sent for the histopathologic examination which revealed the presence of fungus known as "zygomycetes" (invasive zygomycosis).
In this way, this patient suffering from "right side maxillary mucormycosis with osteomyelitis" was successfully managed surgically and was also rehabilitated prosthodontically at M. A. Rangoonwala dental college, Pune.

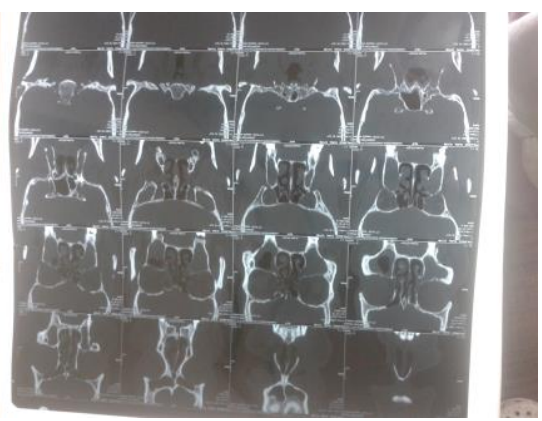

Fig. 1: Radiographic report showing "right maxillary sinusitis"

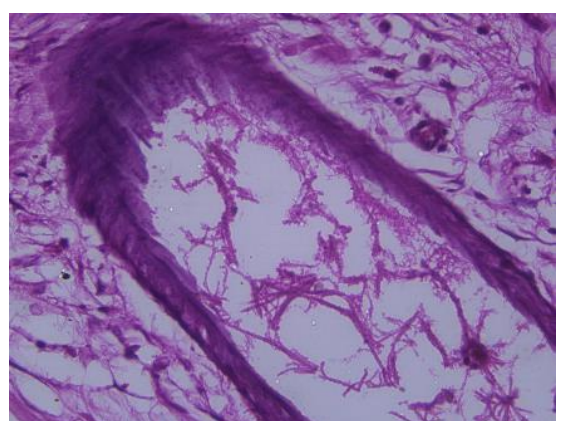

Fig. 2: Histopathological slide image

\section{Discussion}

Mucormycosis is a rapidly progressing angioinvasive fungal infection caused by genera from the order Mucorales. ${ }^{1,4}$ Mucorales and Entomophthorales are two orders of the class Zygomycetes. Patients are predisposed to mucormycosis infection due to 
immunocmoprmising states. Entomophthoramycosis is a chronic subcuataneous infection produced by genera from the order Entomophthorales in immunocompetent patients. Aspergillosis and candidiasis are the most common fungal infection .Mucormycosis is the third most common invasive fungal infection. Out of all fungal infections, $8.3 \%-13 \%$ are represented by mucormycosis. ${ }^{1}$

Pulmonary, rhino-orbito-cerebral/ rhino-cerebral, cutaneous, gastrointestinal and disseminated are major forms of infection which exist. A major risk factor for mucormycosis is the haematological malignancy. Rhinocerebral mucormycosis is the most commonly reported form of "mucormycosis". It is seen more commonly in patients suffering from uncontrolled diabetes mellitus. ${ }^{10}$ Rhinocerebral mucormycosis can be subdivided into rhinomaxillary \& rhino-oculocerebral forms. Rhino-oculocerebral forms are characterized by a high mortality rate. In Leukemic patients, who are receiving chemotherapy, pulmonary mucormycosis is noted. ${ }^{3}$

Sinuses and brain are frequently involved as the primary sites of infection by mucormycosis. ${ }^{4}$ Mucormycosis is identified by fungal invasion of hard palate followed by paranasal sinusus, orbit and then brain. $^{3}$ In a healthy individual, mucormycosis with maxillary sinus is very rare. Symptoms like sinus pain, fever and headache are experienced by patients with affected sinuses. ${ }^{2}$ Arteries are invaded by fungus which leads to thrombus formation in blood vessels resulting in decrease in blood supply followed by tissue necrosis. ${ }^{4}$ Histopathological examination plays an important role in definitive diagnosis of mucormycosis and helps to avoid delay in treatment. ${ }^{10}$

The management of mucormycosis consists of necrotic tissue debridement surgically, accompanied by systemic antifungal therapy. Antifungal therapy consists of administration of systemic high dose amphotericin B. Intravenous liposomal amphotericin and hyperbaric oxygen therapy can also be used.
Funding: No funding sources.

Conflict of interest: None declared.

\section{References}

1. R.M. Prabhu and R. Patel. Mucormycosis and entomophthormycosis: a review of the clinical manifestations, diagnosis and treatment. Clin Microbiol Infect 2004;10(suppl. 1):31-47.

2. Jayashri Anand Khiste, Saroj Ashok Bolde, Gopal Ambadas Pandit, Naveed Abdulsattar Tamboli. Mucormycosis of maxillary sinus in immunocompetent patient masquerading as neoplasm: a case report. Int J Oral Maxillofac Pathol 2013;4(4):50-3.

3. Komali Garlapati, Sunanda Chavva, Rahul Marshal Vaddaswarupu, Jyotsna Surampudi. Fulminant mucormycosis involving paranasal sinuses: a rare case report. Case reports in dentistry, Volume 2014, article ID 465919.

4. Shokouh Taghipour Zahir. Right maxillary sinus mucormycosis with intracranial extension in a 14 -year old male. JSM clinical case reports 2(6):1068.

5. Jaya Garg, S. Sujatha, Atul Garg, S. C. Parija. Nosocomial cutaneous zygomycosis in a patient with diabetic ketoacidosis. Int J Infectious Dis 2009;13(6):508-10.

6. Lunge SB, Sajjan V, Pandit AM, Patil VB. Rhinocerebrocutaneous mucormycosis caused by Mucor species: A rare causation. Indian Dermatol Online J 2015; 6:189-92.

7. Doni BR, Peerapur BV, Thotappa LH, Hippargi SB Sequence of oral manifestations in rhino-maxillary mucormycosis. Indian J Dent Res 2011;22:331-35.

8. Anjana Arora, Bharati A. Patil, Anurag Adepu ,Rose Reynold .Refractory mucormycosis: A possible cause for maxillary necrosis. J Interdiscip Dent 2017;7:65-8.

9. Pooja Aggarwal, Susmita Saxena, Vishal Bansal. Mucormycosis of maxillary sinus. J Oral Maxillofac Pathol 2007;11(2):66-9.

10. Menickan Selvamani, Mandana Donocghue, Shiva Bharani, G. S .Madhushankari. Mucormycosis causing maxillary osteomyelitis. J Nat Sci Bio Med. 2015;6(2):45659.

How to cite the article: Garde J., Bhagwat H., Vadane A., Gaikwad S. Invasive mucormycosis [zygomycosis] and osteomyelitis affecting maxilla: A rare case report. Int Dent J Student's Res 2018;6(3):66-67. 